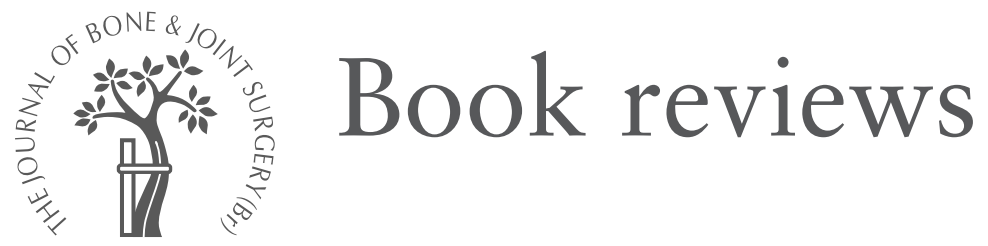

C2004 British Editorial Society of Bone and Joint Surgery doi:10.1302/0301-620X.86B6.15707 \$2.00

J Bone Joint Surg [Br] 2004;86-B:935.

\section{MRI of the hand and wrist}

Edited by Thomas $\mathrm{H}$. Berquist.

Pp 300. Philadelphia: Lippincott Williams and Wilkins, 2003. ISBN: 0-78173796-6. £90

This is a beautifully constructed book covering an area that is tricky to image well. Hand and wrist anatomy is demonstrated by a series of excellent diagrams and top quality MR images, particularly of the soft tissues. The normal MR scans which are shown are useful for anyone trying to learn the cross-sectional anatomy of the hand and wrist. A chapter on imaging technique is useful for technical staff and potential pitfalls and normal variants are covered comprehensively. Further sophisticated techniques such as arthrography and angiography are also included. The subsequent chapters describe the findings in trauma, tumour, arthropathies, infection, avascular necrosis, nerve compression syndromes and a miscellaneous chapter which looked a bit like an excuse to include extraordinary images of the disappearingly rare. Each chapter describes the pathophysiology and the imaging is supported by good diagrams.

My only criticism of the book is that it does not give much advice on when to use MR compared with other imaging methods. For example MR images of osteoarthritis are interesting, but for the vast majority of patients plain radiographs suffice. Conversely, the appearances of psoriasis, gout, ankylosing spondylitis and calcium pyrophosphate deposition are only shown by plain radiography, which begs the question as to why they are included in the book at all.

Although Berquist is listed as the editor he has actually written the vast majority of the chapters himself. As one of the foremost MR authorities he has produced another book which will be excellent for musculoskeletal radiologists and hand surgeons and therapists, but which may be a little too specialised for general orthopaedic interest. I would certainly recommend it as the 'bench book' for the MR units in which I work, and it would be an important addition to an orthopaedic library.

S. Burnett

\section{A computerised pre-clinical test for cemented hip prostheses based on finite element techniques By Jan Stolk}

Pp 191. Netherlands: IFKB, 2002. ISBN: 9-09016-221-6. £25

The author is a biomedical engineer; the book is based on the work he did to gain his $\mathrm{PhD}$. Dr Stolk's research aimed to predict failure of cemented femoral stems by developing a computerised model based on finite element analysis (FEA). Unlike simple structures under simple loading conditions, structural stress analysis for complex geometric forms (e.g. in femoral prostheses) requires the use of more complex FEA techniques.

The model is divided into smaller 'finite elements' of simple geometric form; a computer then balances the forces and moments acting on each individual element and matches these with neighbouring elements. FEA has become widely used in the analysis of the behaviour of prosthetic implants and to predict their long-term behaviour, but to be of value the FEA model should be sufficiently refined to represent the geometry and mechanical behaviour of the implanted prosthesis.

The book consists of ten chapters that clearly describe the various steps undertaken to develop and validate an accurate FEA model. The model was tested by comparing its predictions of behaviour of certain implants with results obtained by experimentally testing the same implants, and with the known clinical outcome of the implants in the Swedish Hip registry. The author should be commended on successfully validating his model by both methods.

The prospect of being able to test new stem designs 'by computer' before they are released onto the market is an encouraging and exciting one. Those predicted to have high rates of failure could then be redesigned or abandoned. One wonders what this model would have predicted for some well-documented failed cemented stems. This interesting and inexpensive book will undoubtedly find appeal within the narrow field of bioengineering and prosthetic design.

Manoj Sood 